Article

\title{
Time-Dependent Motion of a Floating Circular Elastic Plate
}

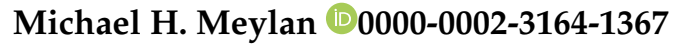 \\ School of Mathematical and Physical Sciences, The University of Newcastle, Callaghan, NSW 2308, \\ Australia;mike.meylan@newcastle.edu.au
}

\begin{abstract}
The motion of a circular elastic plate floating on the surface is investigated in the time-domain. The solution is found from the single frequency solutions and the method to solve for the circular plate is given using the eigenfunction matching method. Simple plane incident waves with a Gaussian profile in wavenumber space are considered and a more complex focused wave group is considered. Results are given for a range of plate and incident wave parameters are investigated. Code is provided to show how to simulate the complex motion.
\end{abstract}

Keywords: Hydroelasticity; Floating Plate; Linear Water Waves

\section{Introduction}

The single frequency solution for the linear water wave problem is extensively used to model the hydroelastic response of a very large floating structures, container ships, or an ice floe [? ? ? ? ]. The simplest example problem in hydroelasticity is the floating elastic plate and this has been the subject of extensive research. Many different method of solution have been developed, including Green function methods [4,5], eigenfunction matching [1,7? ], multi-mode methods [? ] and the Wiener-Hopf method [3? ].

The problem becomes more complicated if we consider the time-dependent problem. If the floating plate is assumed of infinite extent the problem becomes simpler and a spatial Fourier transform gives the solution [10-16]. The forced vibration of a finite floating elastic plate was solved by [17] using a variational formulation and the Rayleigh-Ritz method. The problem was analyzed in shallow water by [21-23] and in finite depth by [? ]. The solution for incident waves in two-dimensions was given in the case of finite depth by [18-20] and for the case of shallow water in two-dimensions by $[21,22]$ and in three-dimensions by [23]. A comparison of two methods for the time-dependent motion in two-dimensions for an initial condition was given in [24]. The solution for finite water depth in three-dimensions was found by [25-28] and was experimentally investigate by [29]. The solution due to a transient incident wave forcing was given in [30]. Recently there has been extensive work on nonlinear simulations using computational fluid dynamics to investigate nonlinear phenomena [? ? ? ? ]. However, even for the case of high amplitude waves the linear wave problem remains valid for a floating plate [? ] and this model continues to the basis of offshore engineering and scattering by ice floe.

The eigenfunction matching method has been applied to many floating elastic plate problems and has proved to give the simplest solutions, provided that the geometry is sufficiently simple that it can be applied. The solution method was first described in [1] and this is where the solution of the special dispersion equation for a floating elastic plate was introduced. This method was extended to circular elastic plates [7], multiple elastic plates [31-33], submerged elastic plates [34,35] and to many other problems.

We present here a solution to the time-dependent problem of floating circular plate subject to incident wave forcing. In part the purpose of this work is to show how simply the complex time-domain motion of such systems can easily be computed using the frequency-domain solution. We also extend the formulation to a Gaussian incident beam. The outline is as follows. In $\S 2$ we derive 
the equations of motion in the time and frequency domain. In $\S 3$ we show how the solution can be found using eigenfunction matching in the frequency domain. In $\$ 4$ we show how the solution in the time domain can be found straightforwardly from the frequency domain solutions.

\section{Equations of Motion}

We consider here a floating elastic plate of uniform thickness and negligible draft. The plate is assumed circular with radius $a$. The fluid is of constant depth $H$ with the $z$ axis pointing vertically up and the free surface at $z=0$. Such a plate has been the subject of extensive research []. The displacement of the plate we denote by $w$ and the velocity potential for the fluid we denote by $\phi$. The equation The plate has uniform thickness $h$. It has been the subject of laboratory experiments [36,37]. It has proved to be a robust model, and it reduces to that of a rigid body in the case of long waves. We begin by stating the governing equations for the plate-water system, which [9] discusses in detail, assuming that the problem is governed by the equation of linear water waves. The kinematic condition is

$$
\partial_{t} w=\partial_{z} \Phi, \quad z=0 ;
$$

where $w$ is the displacement of the fluid surface (which is also the displacement of the plate for $r<a$ ) and $\Phi$ is the velocity potential of the fluid. The dynamic condition is

$$
\rho g w+\rho \partial_{t} \Phi=\left\{\begin{array}{cc}
\frac{E h^{3}}{12\left(1-v^{2}\right)} \partial_{x}^{4} w+\rho_{i} h \partial_{t}^{2} w, \quad r<a, \quad z=0 ; \\
0, \quad r>a
\end{array}\right.
$$

where $\rho$ is the water density, $g$ is the gravitational acceleration, $E$ is the Young's modulus of the plate, $v$ is its Poisson's ratio, and $\rho_{i}$ is its density. Laplace's equation applies throughout the fluid

$$
\Delta \Phi=0,-h<z<0
$$

and the usual non-flow condition at the bottom surface

$$
\partial_{z} \Phi=0, z=-H
$$

Assuming that all motions are time harmonic with radian frequency $\omega$, the velocity potential of the water, $\Phi$, can be expressed as

$$
\Phi(\mathbf{x}, z, t)=\operatorname{Re}\left\{\phi(\mathbf{x}, z) \mathrm{e}^{-\mathrm{i} \omega t}\right\} \quad \text { and } \quad w(\mathbf{x}, t)=\operatorname{Re}\left\{\eta(\mathbf{x}) \mathrm{e}^{-\mathrm{i} \omega t}\right\},
$$

where the reduced velocity potential $\phi$ is complex-valued, and $\mathbf{x}=(x, y)$ is the horizontal spatial variable.

The frequency-domain potential satisfies the boundary value problem

$$
\begin{gathered}
\Delta \phi=0, \quad-H<z<0, \\
\partial_{z} \phi=0, \quad z=-H, \\
\partial_{z} \phi=\alpha \phi, \quad z=0, \mathbf{x} \notin \Omega, \\
\left(\beta \bar{\Delta}^{2}+1-\alpha \gamma\right) \partial_{z} \phi=\alpha \phi, \quad z=0, \mathbf{x} \in \Omega,
\end{gathered}
$$

where $\bar{\Delta}$ is the Laplacian operator in the horizontal plane. The constant $\alpha=\omega^{2} / g$ and $\beta$ and $\gamma$ are

$$
\beta=\frac{E h^{3}}{12\left(1-v^{2}\right) \rho g} \quad \text { and } \quad \gamma=\frac{\rho_{i} h}{\rho},
$$




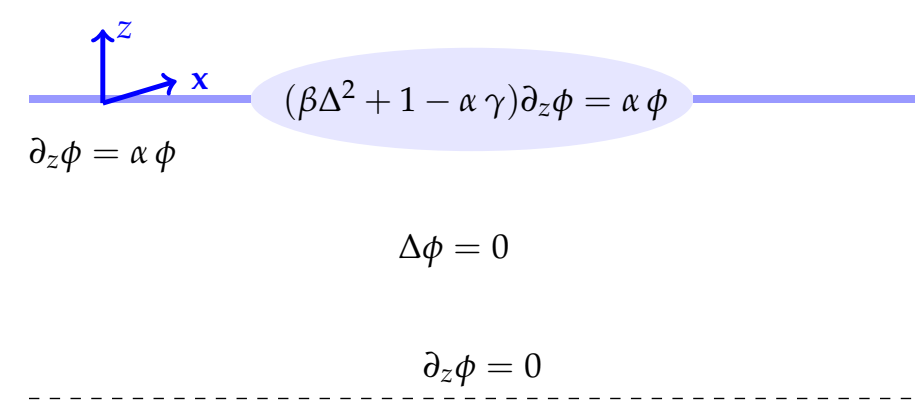

Figure 1. Frequency Domain Equations for a Floating Circular Plate.

The free plate boundary conditions and the radiation condition need to be applied. Figure 1 gives a schematic diagram of the problem.

\section{Eigenfunction Matching}

We derive the solution by the eigenfunction matching method here. The solution in two-dimensions first appeared in [1] and the three-dimensional solution was given in [7]. We begin by separating variables and writing

$$
\phi(\mathbf{x}, z)=\zeta(z) X(\mathbf{x})
$$

Applying Laplace's equation we obtain

$$
\zeta_{z z}+\mu^{2} \zeta=0
$$

so that

$$
\zeta=\cos \mu(z+H),
$$

where the separation constant $\mu^{2}$ must satisfy the standard dispersion equations

$$
\begin{gathered}
k \tan (k H)=-\alpha, \quad \mathbf{x} \notin \Omega, \\
\kappa \tan (\kappa H)=\frac{-\alpha}{\beta \kappa^{4}+1-\alpha \gamma}, \quad \mathbf{x} \in \Omega .
\end{gathered}
$$

Note that we have set $\mu=k$ under the free surface and $\mu=\kappa$ under the plate. The dispersion equations are discussed in detail in [1]. We denote the negative imaginary solution of (11) by $k_{0}$ and the positive real solutions by $k_{m}, m \geq 1$. The solutions of (12) will be denoted by $\kappa_{m}, m \geq-2$. The fully complex solutions with positive real part are $\kappa_{-2}$ and $\kappa_{-1}$ (where $\kappa_{-1}=\overline{\kappa_{-2}}$ ), the negative imaginary solution is $\kappa_{0}$ and the positive real solutions are $\kappa_{m}, m \geq 1$. We define

$$
\phi_{m}(z)=\frac{\cos k_{m}(z+H)}{\cos k_{m} H}, \quad m \geq 0,
$$

as the vertical eigenfunction of the potential in the open water region and

$$
\psi_{m}(z)=\frac{\cos \kappa_{m}(z+H)}{\cos \kappa_{m} H}, \quad m \geq-2,
$$

as the vertical eigenfunction of the potential in the plate covered region.

We now use circular symmetry to write

$$
X(\mathbf{x})=\rho_{n}(r) \mathrm{e}^{\mathrm{i} n \theta}
$$


where $(r, \theta)$ are the polar coordinates in the $\mathbf{x}$ direction. We now solve for the function $\rho_{n}(r)$. Using Laplace's equation in polar coordinates we obtain

$$
\frac{\mathrm{d}^{2} \rho_{n}}{\mathrm{~d} r^{2}}+\frac{1}{r} \frac{\mathrm{d} \rho_{n}}{\mathrm{~d} r}-\left(\frac{n^{2}}{r^{2}}+\mu^{2}\right) \rho_{n}=0,
$$

where $\mu$ is $k_{m}$ or $\kappa_{m}$, depending on whether $r$ is greater or less than $a$. We can convert this equation to the standard form by substituting $y=\mu r$ to obtain

$$
y^{2} \frac{d^{2} \rho_{n}}{\mathrm{~d} y^{2}}+y \frac{\mathrm{d} \rho_{n}}{\mathrm{~d} y}-\left(n^{2}+y^{2}\right) \rho_{n}=0
$$

The solution of this equation is a linear combination of the modified Bessel functions of order $n$, $I_{n}(y)$ and $K_{n}(y)$. Since the solution must be bounded we know that under the plate the solution will be a linear combination of $I_{n}(y)$ while outside the plate the solution will be a linear combination of $K_{n}(y)$. Therefore the potential can be expanded as

$$
\begin{aligned}
& \phi(r, \theta, z)=\sum_{n=-\infty}^{\infty} \sum_{m=0}^{\infty} a_{m n} K_{n}\left(k_{m} r\right) \mathrm{e} \mathrm{e}^{\mathrm{i} n \theta} \phi_{m}(z), \quad r>a, \\
& \phi(r, \theta, z)=\sum_{n=-\infty}^{\infty} \sum_{m=-2}^{\infty} b_{m n} I_{n}\left(\kappa_{m} r\right) \mathrm{e}^{\mathrm{i} n \theta} \psi_{m}(z), \quad r<a,
\end{aligned}
$$

where $a_{m n}$ and $b_{m n}$ are the coefficients of the potential in the open water and the plate covered region respectively.

The incident potential is a wave of amplitude $A$ in displacement travelling in the positive $x$-direction. Following [?] the incident potential can therefore be written as

$$
\phi^{\mathrm{I}}=\frac{A}{\mathrm{i} \sqrt{\alpha}} e^{k_{0} x} \phi_{0}(z)=\sum_{n=-\infty}^{\infty} e_{n} I_{n}\left(k_{0} r\right) \phi_{0}(z) \mathrm{e}^{\mathrm{i} n \theta}
$$

where $e_{n}=A /(\mathrm{i} \sqrt{\alpha})$.

The boundary conditions for the plate also have to be considered. The vertical force and bending moment must vanish, which can be written as

$$
\left[\bar{\Delta}-\frac{1-v}{r}\left(\frac{\partial}{\partial r}+\frac{1}{r} \frac{\partial^{2}}{\partial \theta^{2}}\right)\right] \eta=0
$$

and

$$
\left[\frac{\partial}{\partial r} \bar{\Delta}-\frac{1-v}{r^{2}}\left(\frac{\partial}{\partial r}+\frac{1}{r}\right) \frac{\partial^{2}}{\partial \theta^{2}}\right] \eta=0,
$$

where $w$ is the time-independent surface displacement, $v$ is Poisson's ratio, and $\bar{\Delta}$ is the in polar coordinates is

$$
\bar{\Delta}=\frac{\partial^{2}}{\partial r^{2}}+\frac{1}{r} \frac{\partial}{\partial r}+\frac{1}{r^{2}} \frac{\partial^{2}}{\partial \theta^{2}}
$$

The surface displacement and the water velocity potential at the water surface are linked through the kinematic boundary condition

$$
\phi_{z}=-\mathrm{i} \sqrt{\alpha} \eta \quad, z=0
$$

The relationship between the potential and the surface displacement is

$$
\begin{gathered}
\eta=\mathrm{i} \sqrt{\alpha} \phi, \quad r>a \\
\left(\beta \bar{\Delta}^{2}+1-\alpha \gamma\right) \eta=\mathrm{i} \sqrt{\alpha} \phi, \quad r<a .
\end{gathered}
$$


The surface displacement can also be expanded in eigenfunctions as

$$
\begin{gathered}
\eta(r, \theta)=\sum_{n=-\infty}^{\infty} \sum_{m=0}^{\infty} \mathrm{i} \sqrt{\alpha} a_{m n} K_{n}\left(k_{m} r\right) \mathrm{e}^{\mathrm{i} n \theta}, \quad r>a, \\
\eta(r, \theta)=\sum_{n=-\infty}^{\infty} \sum_{m=-2}^{\infty} \mathrm{i} \sqrt{\alpha}\left(\beta \kappa_{m}^{4}+1-\alpha \gamma\right)^{-1} b_{m n} I_{n}\left(\kappa_{m} r\right) \mathrm{e}^{\mathrm{i} n \theta}, \quad r<a,
\end{gathered}
$$

using the fact that

$$
\bar{\Delta}\left(I_{n}\left(\kappa_{m} r\right) \mathrm{e}^{\mathrm{i} n \theta}\right)=\kappa_{m}^{2} I_{n}\left(\kappa_{m} r\right) \mathrm{e}^{\mathrm{i} n \theta} .
$$

The boundary conditions (21) and (22) can be expressed in terms of the potential using (28). Since the angular modes are uncoupled the conditions apply to each mode, giving

$$
\begin{aligned}
& \sum_{m=-2}^{\infty}\left(\beta \kappa_{m}^{4}+1-\alpha \gamma\right)^{-1} b_{m n} \times \\
& \left(\kappa_{m}^{2} I_{n}\left(\kappa_{m} a\right)-\frac{1-v}{a}\left(\kappa_{m} I_{n}^{\prime}\left(\kappa_{m} a\right)-\frac{n^{2}}{a} I_{n}\left(\kappa_{m} a\right)\right)\right)=0,
\end{aligned}
$$

and

$$
\begin{aligned}
& \sum_{m=-2}^{\infty}\left(\beta \kappa_{m}^{4}+1-\alpha \gamma\right)^{-1} b_{m n} \times \\
& \left(\kappa_{m}^{3} I_{n}^{\prime}\left(\kappa_{m} a\right)+n^{2} \frac{1-v}{a^{2}}\left(\kappa_{m} I_{n}^{\prime}\left(\kappa_{m} a\right)+\frac{1}{a} I_{n}\left(\kappa_{m} a\right)\right)\right)=0 .
\end{aligned}
$$

The potential and its derivative must be continuous across the transition from open water to the plate covered region. Therefore, the potentials and their derivatives at $r=a$ have to be equal. Again we know that this must be true for each angle and we obtain

$$
\begin{aligned}
e_{n} I_{n}\left(k_{0} a\right) \phi_{0}(z)+\sum_{m=0}^{\infty} a_{m n} K_{n}\left(k_{m} a\right) \phi_{m}(z) \\
=\sum_{m=-2}^{\infty} b_{m n} I_{n}\left(\kappa_{m} a\right) \psi_{m}(z)
\end{aligned}
$$

and

$$
\begin{aligned}
e_{n} k_{0} I_{n}^{\prime}\left(k_{0} a\right) \phi_{0}(z)+\sum_{m=0}^{\infty} a_{m n} k_{m} K_{n}^{\prime}\left(k_{m} a\right) \phi_{m}(z) \\
=\sum_{m=-2}^{\infty} b_{m n} \kappa_{m} I_{n}^{\prime}\left(\kappa_{m} a\right) \psi_{m}(z),
\end{aligned}
$$

for each $n$. We solve these equations by multiplying both equations by $\phi_{l}(z)$ and integrating from $-H$ to 0 to obtain

$$
\begin{gathered}
e_{n} I_{n}\left(k_{0} a\right) A_{0} \delta_{0 l}+a_{l n} K_{n}\left(k_{l} a\right) A_{l}=\sum_{m=-2}^{\infty} b_{m n} I_{n}\left(\kappa_{m} a\right) B_{m l} \\
e_{n} k_{0} I_{n}^{\prime}\left(k_{0} a\right) A_{0} \delta_{0 l}+a_{l n} k_{l} K_{n}^{\prime}\left(k_{l} a\right) A_{l} \\
=\sum_{m=-2}^{\infty} b_{m n} \kappa_{m} I_{n}^{\prime}\left(\kappa_{m} a\right) B_{m l},
\end{gathered}
$$


where

$$
\int_{-H}^{0} \phi_{m}(z) \phi_{n}(z) \mathrm{d} z=A_{m} \delta_{m n}
$$

where

$$
A_{m}=\frac{1}{2}\left(\frac{\cos k_{m} H \sin k_{m} H+k_{m} H}{k_{m} \cos ^{2} k_{m} H}\right)
$$

and

$$
\int_{-H}^{0} \phi_{n}(z) \psi_{m}(z) \mathrm{d} z=B_{m n}
$$

where

$$
B_{m n}=\frac{k_{n} \sin k_{n} H \cos \kappa_{m} H-\kappa_{m} \cos k_{n} H \sin \kappa_{m} H}{\left(\cos k_{n} H \cos \kappa_{m} H\right)\left(k_{n}^{2}-\kappa_{m}^{2}\right)}
$$

Equation (33) can be solved for the open water coefficients $a_{m n}$

$$
a_{l n}=-e_{n} \frac{I_{n}\left(k_{0} a\right)}{K_{n}\left(k_{0} a\right)} \delta_{0 l}+\sum_{m=-2}^{\infty} b_{m n} \frac{I_{n}\left(\kappa_{m} a\right) B_{m l}}{K_{n}\left(k_{l} a\right) A_{l}},
$$

which can then be substituted into equation (34) to give us

$$
\begin{aligned}
& \left(k_{0} I_{n}^{\prime}\left(k_{0} a\right)-k_{0} \frac{K_{n}^{\prime}\left(k_{0} a\right)}{K_{n}\left(k_{0} a\right)} I_{n}\left(k_{0} a\right)\right) e_{n} A_{0} \delta_{0 l} \\
& =\sum_{m=-2}^{\infty}\left(\kappa_{m} I_{n}^{\prime}\left(\kappa_{m} a\right)-k_{l} \frac{K_{n}^{\prime}\left(k_{l} a\right)}{K_{n}\left(k_{l} a\right)} I_{n}\left(\kappa_{m} a\right)\right) B_{m l} b_{m n},
\end{aligned}
$$

for each $n$. Together with equations (29) and (30) equation (40) gives the required equations to solve for the coefficients of the water velocity potential in the plate covered region. For the numerical solution we truncate the sum at $N$ and then we have $N+1$ equations from matching through the depth and 2 extra equations from the boundary conditions.

It should be noted that the solutions for positive and negative $n$ are complex conjugates so that they do not both need to be calculated. There are some minor simplifications which are a consequence of this which are discussed in more detail in [? ].

\section{Time-dependent forcing and numerical results}

We have denoted the surface displacement in the frequency domain is given by $\eta(\mathbf{x})$. However the surface displacement is a function of $\omega$ and $\omega$ is in turn a function of wavenumber $k$. We have also only considered waves incident from the positive $x$ direction $(\theta=0)$. This makes sense given the circular symmetry, but we can consider waves incident from other angles (which are found by rotation of the solution by the angle). Therefore we denote the complex frequency domain surface displacement by $\eta((x), \theta, k)$.

\subsection{Plane incident wave forcing}

The simplest time-dependent problem is to consider a place incident wave from the positive $x$ direction. We assume that the incident wave is a Gaussian at $t=0$. Therefore the time-dependent displacement is then given by the following Fourier integral

$$
w(\mathbf{x}, t)=\operatorname{Re}\left\{\int_{0}^{\infty} \hat{f}(k) \eta(\mathbf{x}, 0, k) \mathrm{e}^{\mathrm{i} \omega t} \mathrm{~d} k\right\},
$$

where $\hat{f}(k)$ is

$$
\hat{f}(k)=\sqrt{\frac{\sigma}{\pi}} \exp \left(\sigma\left(k-k_{0}\right)^{2}\right) .
$$



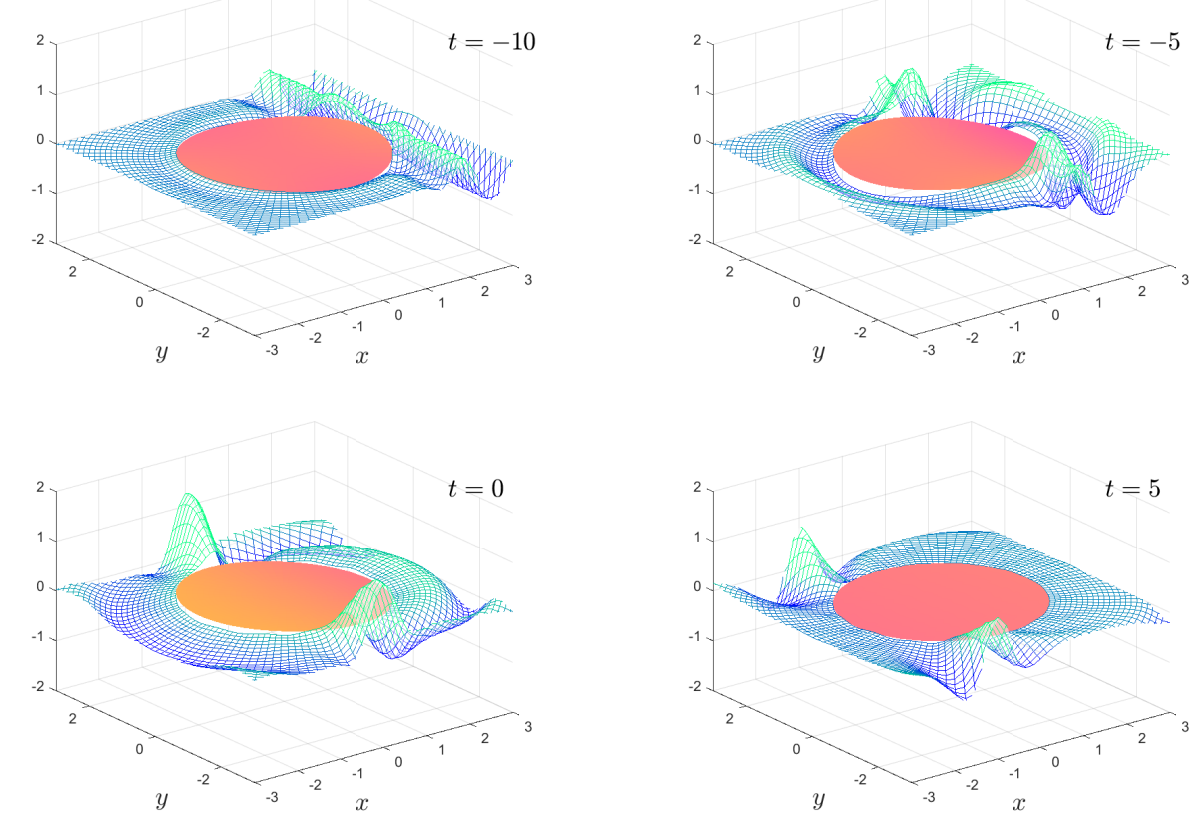

Figure 2. The time-dependent motion $\beta=1 \times 10^{-1}, \gamma=0, h=1$ and $a=2$ for the times shown. The full animation can be found in movie 1 .

where $\sigma$ is a scale factor and we set $\sigma-0.1$ and $k_{0}$ is the central wavenumber we set $k_{0}=3$.

\subsection{Focused wave group}

It is more interesting to consider a two-dimensional incident waves. A simple focused wave group can be constructed as from the following formula

$$
w(\mathbf{x}, t)=\operatorname{Re}\left\{\int_{-\pi / 2}^{\pi / 2} \int_{0}^{\infty} \hat{f}(k) \mathrm{e}^{-\theta_{\sigma} k^{2} \sin ^{2}(\theta)} \eta(\mathbf{x}, \theta, k) \mathrm{e}^{\mathrm{i} \omega t} \mathrm{~d} k \mathrm{~d} \theta\right\},
$$

where $\theta_{\sigma}$ is another scaling parameters which we set to be $\theta_{\sigma}=0.1$

The numerical results we present are a subset of the possible motions which are possible. We fix the mass $\gamma=0$, the water depth $h=1$ and the floe radius $a=2$ for all calculations. The solution is shown as an animation in movies 1 to 8 which are given as supplementary material. Figures 2 to 5 show snapshots from movies 1 to 4 respectively for the times $t=-5,0,5,10$. We change the stiffness from $\beta=1 \times 10^{-1}$ in Figure 2to $\beta=1 \times 10^{-4}$ in Figure 5 . The plate goes from being virtually stiff to highly flexible. The complex motion of the plate and fluid systems can be seen, especially in the movies in the supplementary material.

Figures 6 to 9 show the solution for the more complicated and interesting case of an incident wave packet. The complex and resonant behaviour of the plate and fluid system is clear visible.

\section{Conclusions}

The purpose of this work is to show how we can easily visualise the complex time-domain behaviour of complex wave scattering problems such as those which arise from the scattering by a flexible plate. While the frequency-domain solution is central to our calculations, the scattering results from the frequency domain solution are often difficult to interpret in the context of incident wave packets. By the simple visualisation using the suitable superposition of incident waves we can bring the complex motion to life. The authors hopes that these results, and the accompanying computer code, 

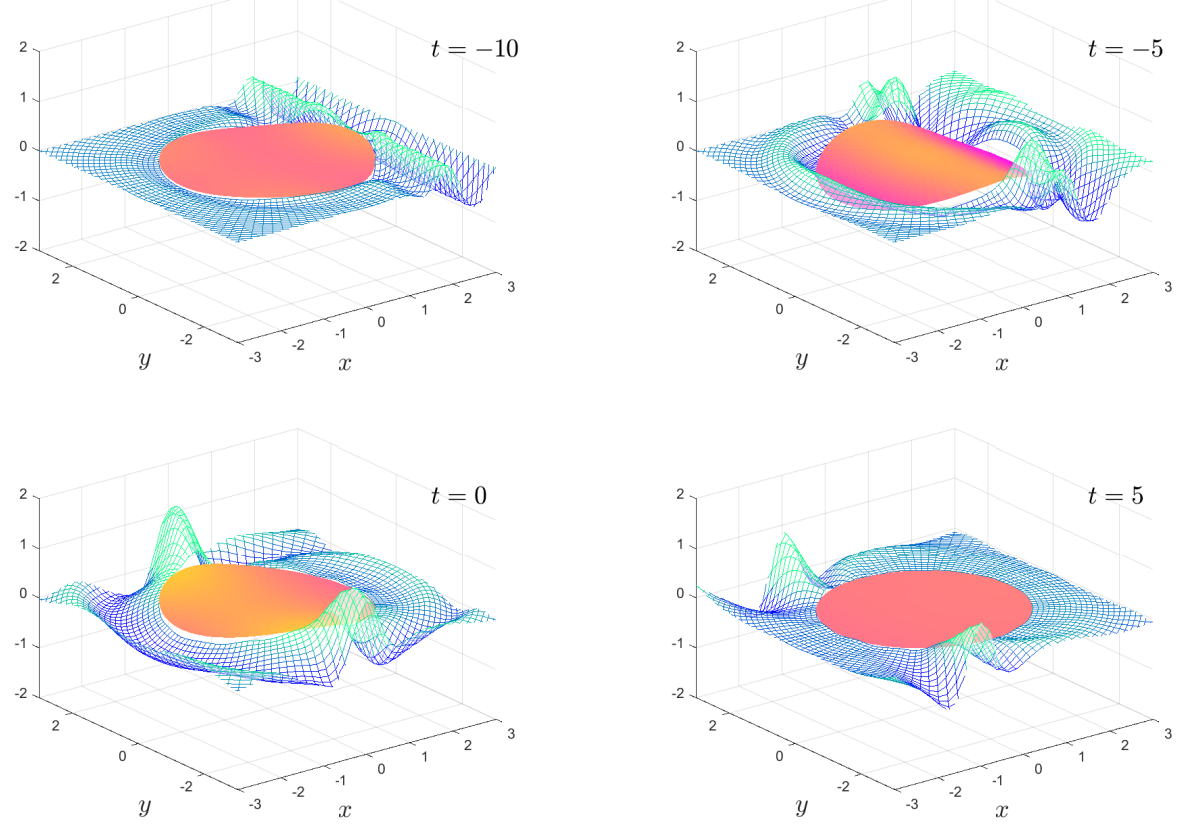

Figure 3. As in Figure 2 except $\beta=1 \times 10^{-2}$. The full animation can be found in movie 2 .
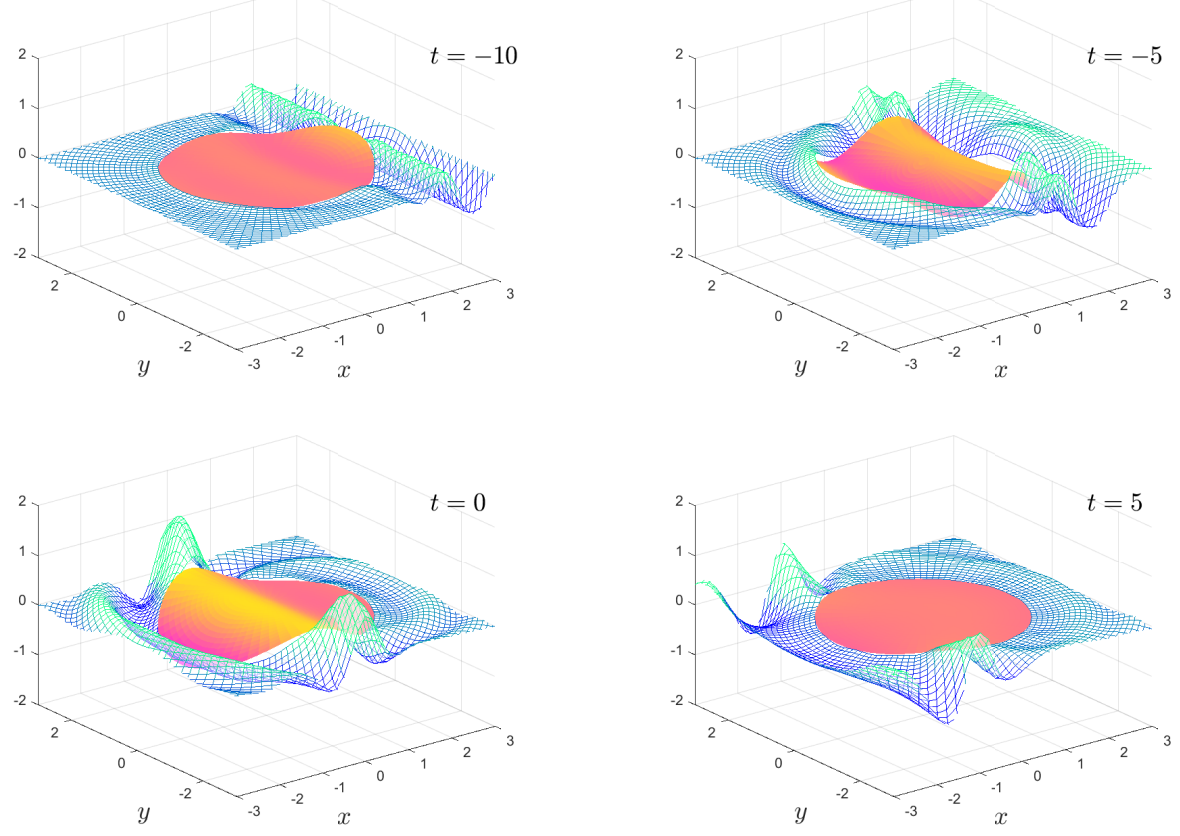

Figure 4. As in Figure 2 except $\beta=1 \times 10^{-3}$. The full animation can be found in movie 3 . 

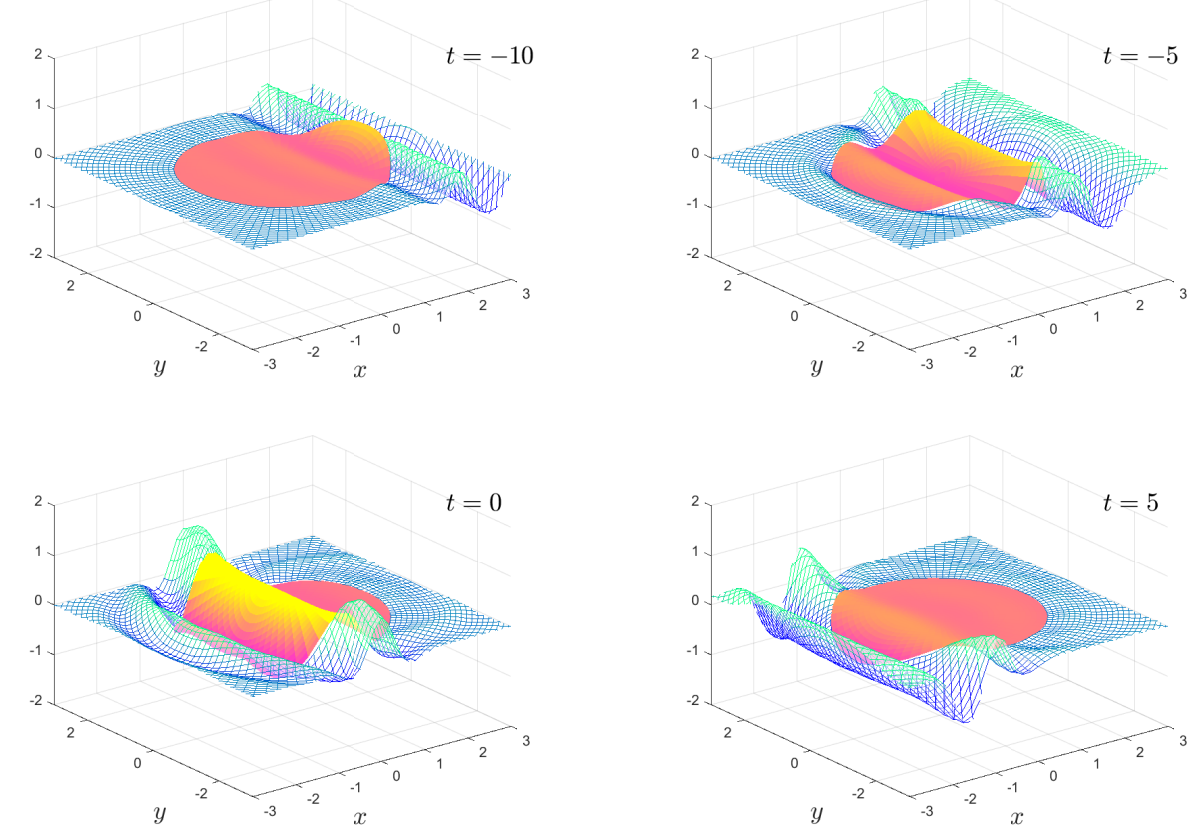

Figure 5. As in Figure 2 except $\beta=1 \times 10^{-4}$. The full animation can be found in movie 4 .
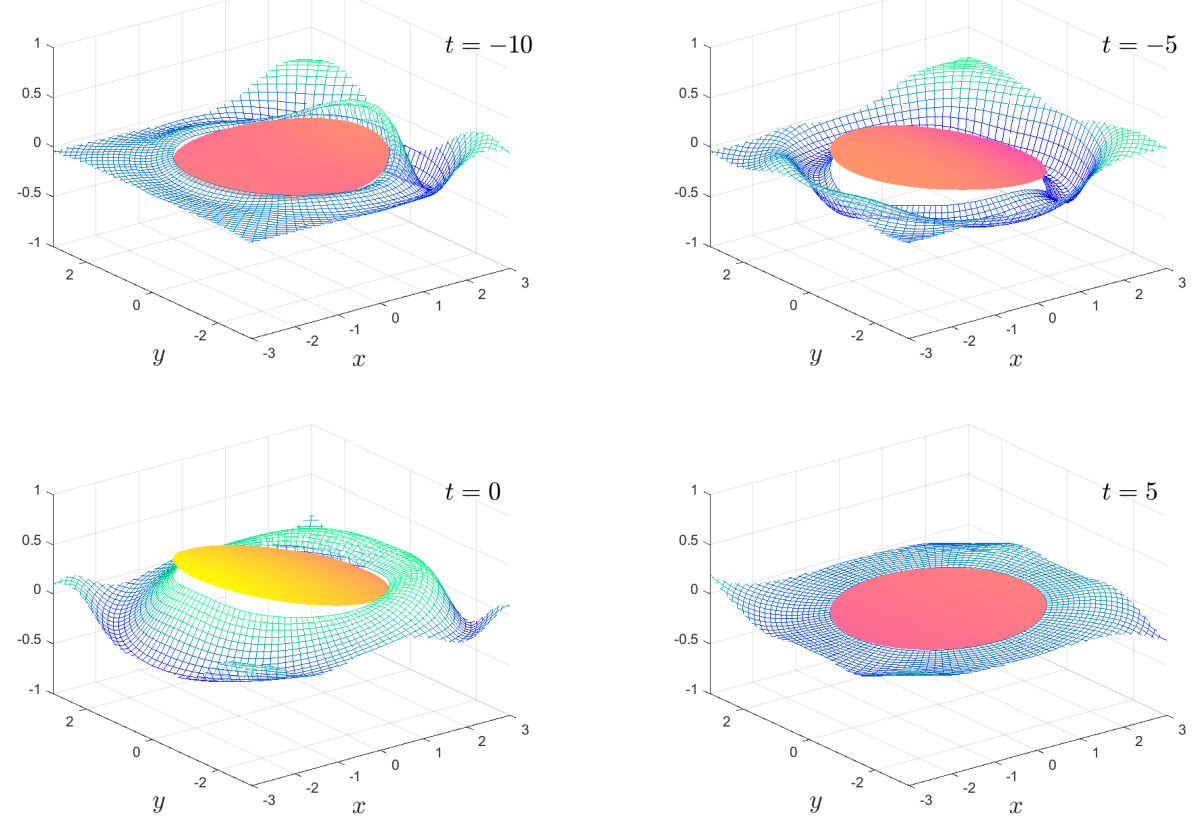

Figure 6. The time-dependent motion $\beta=1 \times 10^{-1}, \gamma=0, h=1$ and $a=2$ for the times shown. The full animation can be found in movie 5 . 

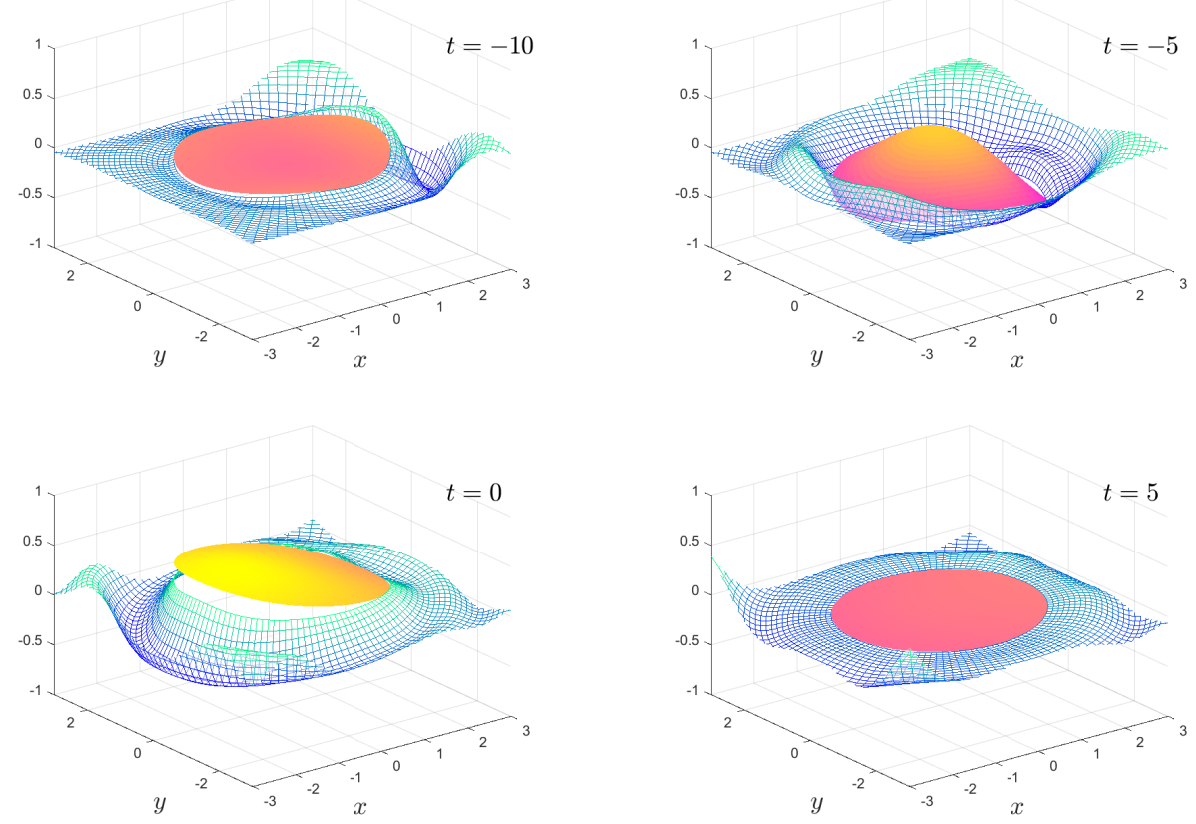

Figure 7. As in Figure 6 except $\beta=1 \times 10^{-3}$. The full animation can be found in movie 6 .
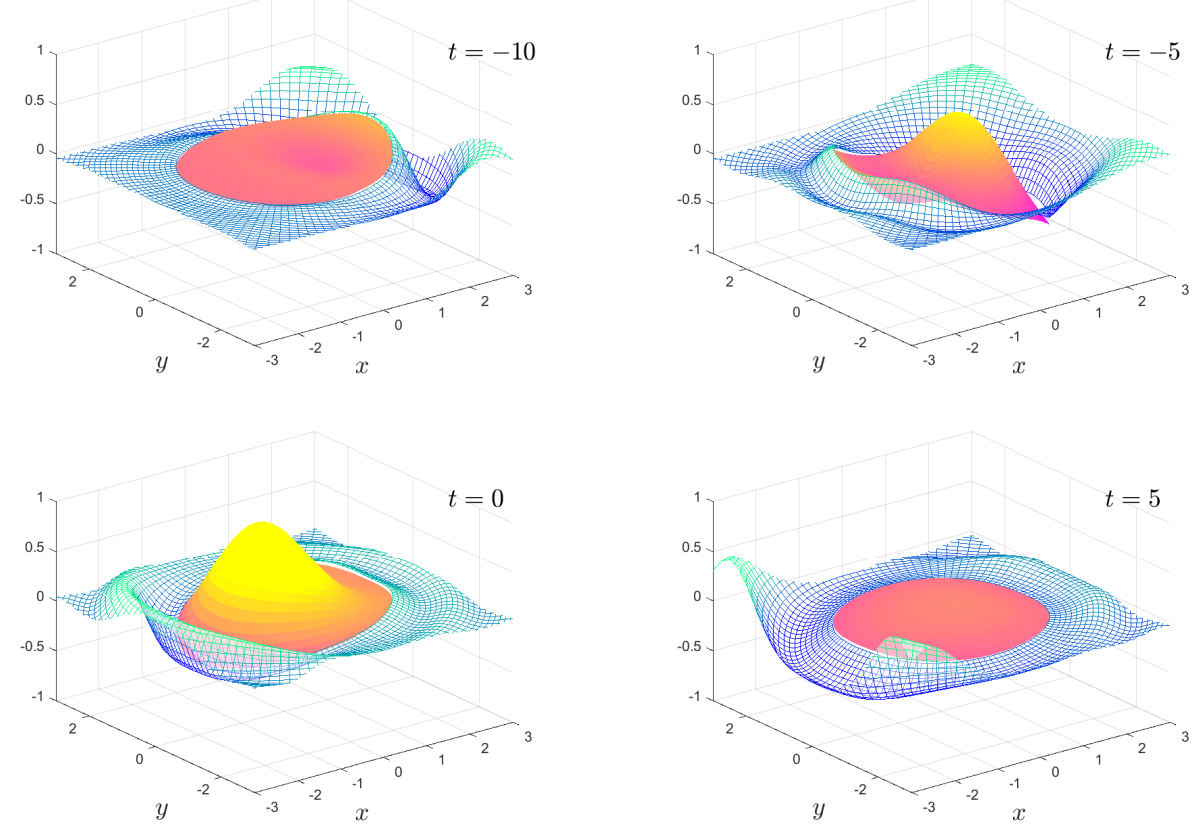

Figure 8. As in Figure 6 except $\beta=1 \times 10^{-4}$. The full animation can be found in movie 7 . 

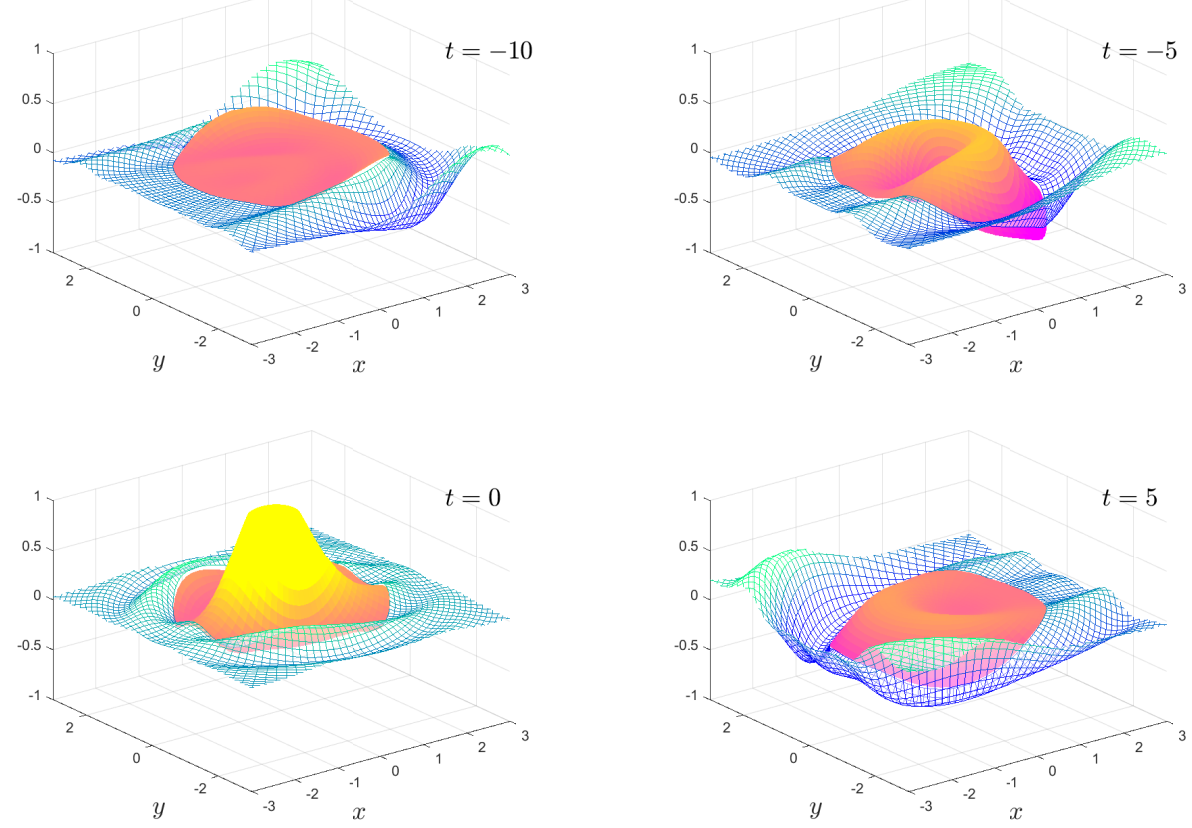

Figure 9. As in Figure 6 except $\beta=1 \times 10^{-2}$. The full animation can be found in movie 8 .

will encourage others to also investigate such visualisations for their complex wave wave scattering problem.

\section{Acknowledgments}

This work is funded by the Australian Research Council (DP200102828).

1. Fox, C.; Squire, V.A. On the Oblique Reflexion and Transmission of Ocean Waves at Shore Fast Sea Ice. Phil. Trans. R. Soc. Lond. A. 1994, 347, 185-218.

2. Sahoo, T.; Yip, T.L.; Chwang, A.T. Scattering of surface waves by a semi-infinite floating elastic plate. Physics of Fluids 2001, 13, 3215-3222.

3. Chung, H.; Fox, C. Calculation of wave-ice interaction using the Wiener-Hopf technique. New Zealand J. Math. 2002, 31, 1-18.

4. Meylan, M.H.; Squire, V.A. The Response of Ice Floes to Ocean Waves. J. Geophy. Res. 1994, 99, 891-900.

5. Newman, J.N. Wave effects on deformable bodies. Appl. Ocean Res. 1994, 16, 45 - 101.

6. Meylan, M.H.; Squire, V.A. Response of a Circular Ice Floe to Ocean Waves. J. Geophys. Res. 1996, 101, 8869-8884.

7. Peter, M.A.; Meylan, M.H.; Chung, H. Wave scattering by a circular elastic plate in water of finite depth: a closed form solution. Int. J. Offshore Polar 2004, 14, 81-85.

8. Kashiwagi, M. A B-Spline Galerkin Scheme for Calculating Hydroelastic Response of a Very Large Floating Structure in Waves. J. Marine Sci. and Tech. 1998, 3, 37-49.

9. Meylan, M.H. The Wave Response of Ice Floes of Arbitrary Geometry. J. Geophys. Res. 2002, 107.

10. Davys, J.W.; Hosking, R.J.; Sneyd, A.D. Waves due to a Steadily Moving Source on a Floating Ice Plate. J. Fluid Mech. 1985, 158, 269-287.

11. Hosking, R.J.; Sneyd, A.D.; Waugh, D.W. Viscoelastic Response of a Floating Ice Plate to a Steadily Moving Load. J. Fluid Mech. 1988, 196, 409-430.

12. Milinazzo, F.; Shinbrot, M.; Evans, N.W. A Mathematical Analysis of the Steady Response of Floating Ice to the Uniform Motion of a Rectangular Load. J. Fluid Mech. 1995, 287, 173-197.

13. Squire, V.A.; Hosking, R.J.; Kerr, A.D.; Langhorne, P.J. Moving Loads on Ice Plates; Kluwer, 1996. 
14. Nugroho, W.; Wang, K.; Hosking, R.; Milinazzo, F. Time-dependent response of a floating flexible plate to an impulsively started steadily moving load. J. Fluid Mech. 1999, 381, 337-355.

15. Wang, K.; Hosking, R.; Milinazzo, F. Time-dependent response of a floating viscoelastic plate to an impulsively started moving load. J. Fluid Mech. 2004, 521, 295-317.

16. Bonnefoy, F.; Meylan, M.; Ferrant, P. Nonlinear higher-order spectral solution for a two-dimensional moving load on ice. J. Fluid Mech. 2009, 621, 215-242.

17. Meylan, M.H. The forced vibration of a thin plate floating on an infinite liquid. J. Sound Vib. 1997, 205, 581-591.

18. Tkacheva, L. Plane problem of vibrations of an elastic floating plate under periodic external loading. Journal of applied mechanics and technical physics 2004, 45, 420-427.

19. Tkacheva, L. Action of a periodic load on an elastic floating plate. Fluid Dynamics 2005, 40, $282-296$.

20. Hazard, C.; Meylan, M.H. Spectral theory for a two-dimensional elastic thin plate floating on water of finite depth. SIAM J. Appl. Math. 2007, 68, 629-647.

21. Meylan, M.H. Spectral Solution of Time Dependent Shallow Water Hydroelasticity. J. Fluid Mech. 2002, 454, 387 - 402.

22. Sturova, I.V. Unsteady behavior of an elastic beam floating on shallow water under external loading. J. Appl. Mech. and Tech. Phys. 2002, 43, 415-423.

23. Sturova, I.V. The action of an unsteady external load on a circular elastic plate floating on shallow water. J. Appl. Maths Mechs. 2003, 67, 407-416.

24. Meylan, M.H.; Sturova, I.V. Time-Dependent Motion of a Two-Dimensional Floating Elastic Plate. J. Fluid. Struct. 2009, 25, 445-460.

25. Kashiwagi, M. A Time-Domain Mode-Expansion Method for Calculating Transient Elastic Responses of a Pontoon-Type VLFS. J. of Marine Sci. and Tech. 2000, 5, 89-100.

26. Kashiwagi, M. Transient responses of a VLFS during landing and take-off of an airplane. J. of Marine Sci. and Tech. 2004, 9, 14-23.

27. Qui, L. Numerical simulation of transient hydroelastic response of a floating beam induced by landing loads. Appl. Ocean Res. 2007, 29, 91-98.

28. Matiushina, A.A.; Pogorelova, A.V.; Kozin, V.M.; others. Effect of Impact Load on the Ice Cover During the Landing of an Airplane. International Journal of Offshore and Polar Engineering 2016, 26, 6-12.

29. Endo, H.; Yago, K. Time history response of a large floating structure subjected to dynamic load. Journal of the Society of Naval Architects of Japan 1999, 1999, 369-376.

30. Montiel, F.; Bennetts, L.; Squire, V. The transient response of floating elastic plates to wavemaker forcing in two dimensions. Journal of Fluids and Structures 2012, 28, 416-433.

31. Kohout, A.L.; Meylan, M.H. Wave Scattering by Multiple Floating Elastic Plates with Spring or Hinged Boundary Conditions. Mar. Struct. 2009, 22, 712-729.

32. Kohout, A.L.; Meylan, M.H. An elastic plate model for wave attenuation and ice floe breaking in the marginal ice zone. J. Geophys. Res. 2008, 113.

33. Kohout, A.L.; Meylan, M.H.; Sakai, S.; Hanai, K.; Leman, P.; Brossard, D. Linear Water Wave Propagation Through Multiple Floating Elastic Plates of Variable Properties. J. Fluid. Struct. 2007, 23, 649-663.

34. Mahmood-ul-Hassan,.; Meylan, M.H.; Peter, M.A. Water-Wave Scattering by Submerged Elastic Plates. Quart. J. Mech. Appl. Math. 2009, 62, 321-344.

35. Behera, H.; Sahoo, T. Hydroelastic analysis of gravity wave interaction with submerged horizontal flexible porous plate. Journal of Fluids and Structures 2015, 54, 643-660.

36. Montiel, F.; Bennetts, L.G.; Squire, V.A.; Bonnefoy, F.; Ferrant, P. Hydroelastic response of floating elastic discs to regular waves. Part 1. Wave basin experiments. J. Fluid Mech. 2013, 723, 604-628.

37. Montiel, F.; Bennetts, L.G.; Squire, V.A.; Bonnefoy, F.; Ferrant, P. Hydroelastic response of floating elastic discs to regular waves. Part 1. Modal analysis. J. Fluid Mech. 2013, 723, 629-652. 